\title{
Editorial für die Zeitschrift für Orthopädie und Unfallchirurgie
}

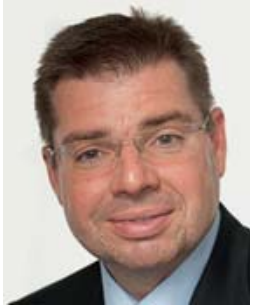

Dieter C. Wirtz

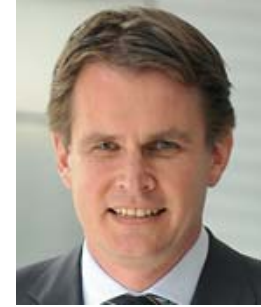

Ulrich Stöckle

Korrespondenzadressen

Univ.-Prof. Dr. med. Dieter C. Wirtz

Direktor der Klinik und Poliklinik für Orthopädie und

Unfallchirurgie

Universitätsklinikum Bonn

Sigmund-Freud-Straße 25, 53105 Bonn

Tel.: 02 28/287-14170, Fax: 02 28/287-14175

dieter.wirtz@ukb.uni-bonn.de

Univ.-Prof. Dr. Ulrich Stöckle

Ärztlicher Direktor

BGU Klinik Tübingen

Schnarrenbergstraße 96, 72076 Tübingen

Tel.: 07071/606-1001, Fax: 0 7071/606-1002

ustoeckle@bgu-tuebingen.de

Bibliografie

DOI https://doi.org/10.1055/s-0043-102976

Z Orthop Unfall 2017; 155: 271-272 @ Georg Thieme

Verlag KG Stuttgart · New York | ISSN 1864-6697

\section{Liebe Leserinnen, liebe Leser,}

auch in der aktuellen Ausgabe unserer Zeitschrift haben wir wieder ein breitgefächertes Themenfeld für Sie zusammengestellt und hoffen, dass Sie interessante Anregungen für Ihren klinischen Alltag finden können. Dabei ist es uns besonders wichtig, neben Übersichtsarbeiten insbesondere auch wissenschaftliche Originalarbeiten zu publizieren, die neue Erkenntnisse und Innovationen unseres Faches beinhalten.

In der Übersichtsarbeit „Osteoimmunologie - immunologische Einflüsse auf den Knochenauf- und abbau“ [1] stellen die Autoren Limmer und Wirtz den aktuellen Erkenntnisstand der Osteoimmunologie dar. Dabei werden neue Ansätze zur immunologischen Beeinflussung der Knochendefektregeneration dargestellt und perspektivisch die Nutzung von immunmodulatorischen Medikamenten zur Behandlung ossärer Erkrankungen diskutiert.

Bornemann et al. [2] analysieren im 2. Übersichtsartikel pathologische Veränderungen im Iliosakralgelenk als Genese tieflumbaler-glutealer Schmerzen. Abgeleitet von klinischen und bildgebenden Diagnostikprinzipien werden die ersten Erfahrungen eines neuen, das Iliosakralgelenk versteifenden Implantates (iFUSE) vorgestellt.

In der 3. Übersichtsarbeit widmen sich Schwarze et al. [3] der „Gutachterlichen Beurteilung des traumatischen Bandscheibenvorfalls". Dabei wird hervorgehoben, dass die exakte Unfallanamnese, der Beschwerdeverlauf, die Analyse konkurriender Ursachen und insbesondere das unfallnahe MRT zur Sicherung des Körpererstschadens überragende Bedeutung haben. In diesem Zusammenhang wird zur Kausalitätsprüfung von den Autoren ein Prüfschema vorgestellt, das den Begutachtungsprozess über Einstiegs-, Realisierungs- und Entscheidungsebene hinweg unterstützt.

Aufgrund der zunehmenden Behandlung von Verletzten aus Konfliktländern mit Wunden mit multiresistenten Erregern kommt es zu einem veränderten Keimspektrum von Komplexwunden in Deutschland. Hierbei zeigen Riesner et al. [4] in einer zusammenfassenden Originalarbeit die aktuelle Keimsituation in Deutschland in den vergangen 10 Jahren auf und berichten über Erfahrungen mit der Behandlung von Verwundeten aus Kriegszonen.

Ebenfalls mit multiresistenten Erregern beschäftigt sich die Gruppe um Maier et al. [5]. Sie analysierten in einer prospektiven Studie die Durchseuchungsrate eines elektiven orthopädischen Patientenkollektivs mit Livestock-associated MRSA. Da durchaus bei bestimmten Risikogruppen eine erhöhte Kolonisierungsrate besteht, ist ein präoperatives Screening eindeutig zu empfehlen.

In einer weiteren Originalarbeit wurde durch Langmair et al. [6] das diagnostische und therapeutische Vorgehen bei infizierten Hüftgelenkstotalendoprothesen in 107 deutschen Krankenhäusern in einer Onlineumfrage erfragt und ausgewertet. Dabei zeigte sich ein inhomogenes intra- und interklinisches Vorgehen. Es erscheint daher gerade bei diesem - auch aus ökonomischer Sicht - komplexen Patientenkollektiv wichtig, evidenzbasierte Behandlungsempfehlungen festzulegen. 
Einem gänzlich anderen Themenfeld widmet sich die letzte in dieser Ausgabe veröffentlichte Originalarbeit. Meyer et al. [7] untersuchten in einer experimentellen Studie den Einfluss osteoligamentärer Verletzungen auf die Stabilität des atlantoaxialen Komplexes. Dabei zeigte sich, dass neben dem Dens axis insbesondere das Ligamentum transversum eine große Bedeutung für die Stabilität der oberen HWS-Region hat und deshalb bei entsprechenden Verletzungen eine operative Stabilisierung und/oder eine längerfristige Immobilisierung mittels rigider Orthese zu empfehlen ist.

"Abgerundet“ wird das Heft mit 2 sehr interessanten Fallberichten, einem klinischen Erfahrungsbericht von Zwipp et al. [8] zur Nachbehandlung von Rückfußfrakturen mit einem Rückfußentlastungsstiefels der „2. Generation“ und einer aktuellen CME-Fortbildung mit dem Thema der intraartikulären Tibiakopffraktur [9].

Uns hat das Lesen der Artikel Spass gemacht; ein Erkenntnisgewinn ist praktisch immer garantiert. Wir hoffen, dass es Ihnen genauso gehen wird.

Ihre

Dieter C. Wirtz

Ulrich Stoeckle

\section{Literatur}

[1] Limmer A, Wirtz DC. Osteoimmunologie - immunologische Einflüsse auf den Knochenauf- und abbau. Z Orthop Unfall 2017; 155: 273-280

[2] Bornemann R, Pflugmacher R, Koch EMW et al. Moderne Diagnostik und minimalinvasive Operationsmethoden bei Patienten mit schmerzhaftem Iliosakralgelenksyndrom. Z Orthop Unfall 2017; 155: 281-287

[3] Schwarze M, Weber M-A, Bucur FM et al. Gutachtliche Beurteilung des traumatischen Bandscheibenvorfalls. Z Orthop Unfall 2017; 155: 288296

[4] Riesner HJ, Friement B, Lang P et al. Verändertes Keimspektrum von Komplexwunden in Deutschland durch die Behandlung von Verletzten aus Konfliktländern. Z Orthop Unfall 2017; 155: 297-303

[5] Maier GS, Thorey F, Kolbow K et al. Livestock-assoziierter Methicillin-resistenter Staphylococcus aureus - Erhebung einer orthopädischen Fachklinik im Hochrisikogebiet Nord-West. Z Orthop Unfall 2017; 155: 304309

[6] Langmair ER, Konstantinidis L, Südkamp NP et al. Die infizierte Hüfttotalendoprothese: Eine Onlineumfrage zum aktuellen diagnostischen und therapeutischen Vorgehen in 107 deutschen Krankenhäusern. Z Orthop Unfall 2017; 155: 310-317

[7] Meyer C, Bredow J, Heising E et al. Einfluss osteoligamentärer Verletzungen auf die Stabilität des atlantoaxialen Komplexes. Z Orthop Unfall 2017; 155: 318-323

[8] Zwipp H, Borrmann M, Walter E. Erfahrungen mit dem Rückfußentlastungsstiefel. Z Orthop Unfall 2017; 155: 33-339

[9] Reul M, Nijs S, Rommens PM et al. Intraartikuläre Tibiakopffrakturen. Z Orthop Unfall 2017; 155: 352-370 